\title{
LA EXTRAÑEZA DE LAS PALABRAS MÁS FAMILIARES
}

\author{
THE UNCANNINESS OF ORDINARY WORDS
}

\section{Cristina Corredor}

Stanley Cavell, ¿Debemos querer decir lo que decimos? Zaragoza: Prensas Universitarias de Zaragoza, 2018. Traducción de Diego Ribes Nicolás.

En las páginas introductorias del compendio de ensayos ¿Debemos querer decir lo que decimos? (primera edición), Stanley Cavell destaca su deuda con Thompson Clarke por haberle enseñado a entender la epistemología tradicional y, en particular, el escepticismo. Recuerda también que junto a Clarke impartió, entre 1959 y 1960, un seminario sobre las Investigaciones Filosóficas de Wittgenstein. Su defensa de la filosofía del lenguaje ordinario, la profunda influencia de su estudio del último Wittgenstein, y su aproximación al escepticismo como forma de estar en el mundo, recorren la filosofía y la obra de Cavell y se encuentran muy presentes en el conjunto de escritos que componen el volumen que ahora reseñamos. Puede afirmarse que el conjunto de estos diez trabajos, escritos entre 1958 y 1969, muestran ya con claridad la posición filosófica de Cavell y su personal estilo de escritura, lo que en su caso equivale a decir su personal estilo de pensamiento.

La riqueza de los temas que trata el volumen, unida a la complejidad de algunos de los análisis y discusiones, hacen imposible dar cuenta aquí de todo ello con suficiente claridad y detalle. Esta reseña sólo pretenderá dar una idea del conjunto tratando de identificar algunos aspectos característicos y originales del pensamiento y el estilo de Cavell, centrando la atención en dos de sus ensayos más representativos (capítulos I y II). Sólo al final se ofrecerá una reflexión más crítica en diálogo con su planteamiento.

Dos de los ensayos pueden considerarse de crítica literaria: su lectura de Fin de partida, la obra de Beckett (en el ensayo Final del juego de la espera, capítulo V), y su estudio sobre El rey Lear de Shakespeare (en La evitación del amor, capítulo X). Cavell se anticipa a una visión prejuiciada que no consideraría este tipo de estudios críticos prima facie filosofía, señalando la cercanía entre ambas formas de actividad. Otros dos ensayos, Música descompuesta (capítulo 8) y Una cuestión de querer decirlo (capítulo 9), entran en el terreno de la crítica musical y los problemas de interpre- 
tación de la obra artística. En todos estos casos, el método de Cavell consiste en una aplicación original y actualizada de la filosofía del lenguaje ordinario. A través del análisis de las expresiones que se utilizan para la interpretación y la crítica (por ejemplo, preguntando: ¿qué significa una determinada expresión cuando es relativa a la crítica del arte?), Cavell logra hacer evidentes formas inauténticas de expresar la subjetividad y el propio yo, lo que con frecuencia, en su consideración, ocurre inadvertidamente. En todos estos casos se pone de manifiesto su profundo conocimiento de la cultura contemporánea, junto a un conjunto de preocupaciones intelectuales que están presentes así mismo en sus ensayos más académicamente filosóficos.

Estas preocupaciones atañen a la relación entre la modernidad y la tradición, tanto en filosofía como fuera de ella (arte, música, literatura), en la medida en que esa relación se ha vuelto problemática. Y, en directa conexión con ello, atañen a la relación entre las prácticas contemporáneas y la historia de esas prácticas. En el diagnóstico de Cavell, el rechazo al pasado que se pone de manifiesto en el último Wittgenstein (y, apunta también, en la obra Ser y Tiempo de Heidegger) tendría una significación transformada: sería una forma de reconocimiento de que las propias prácticas sólo adquieren identidad sobre el trasfondo de una experiencia continua del pasado. Cavell encuentra un precedente para esta actitud en Kant, por su reconocimiento explícito de que los términos en los que se valora el pasado son específicos de la propia posición presente y requieren de justificación desde esa misma posición.

La interrelación del último Wittgenstein con Kant no acaba, para Cavell, en esta observación. En su Prefacio de 2001 a la reedición del libro, accesible a través de la traducción al castellano que ahora reseñamos, hace completamente explícita su convicción de que es posible entender la apelación filosófica a lo ordinario en relación con la lógica trascendental de Kant, y de que es posible leer así también al último Wittgenstein. La tesis de Cavell afirma que la búsqueda filosófica de Wittgenstein se orienta a identificar las condiciones necesarias del mundo compartido. Encuentra respaldo para este original y personalísimo punto de vista en declaraciones como la que es posible leer en las Investigaciones Filosóficas: "nuestra investigación $(. .$.$) se dirige no hacia los fenómenos, sino, como podría decirse, hacia$ las 'posibilidades' de los fenómenos". (\$90) Aunque la conexión entre esta tesis y el método del análisis del lenguaje ordinario no es evidente, puede decirse que constituye el núcleo del respaldo argumentativo que Cavell es capaz de ofrecer.

Los dos primeros ensayos recogidos en el volumen, ¿Debemos querer decir lo que decimos? (que da título al conjunto) y La accesibilidad de la segunda filosofía de Wittgenstein, desarrollan la tesis arriba mencionada y otras ideas de la personalísima 
posición intelectual de Cavell. En lo sucesivo, esta reseña se centrará en estos dos ensayos, tratando también de indicar las ideas originales y valiosas que pueden encontrarse en los restantes trabajos.

En una aproximación al tema que va a ser constante en su obra y que caracteriza el estilo de pensamiento de Cavell, el ensayo ¿Debemos querer decir lo que decimos? (el más extenso del volumen) comienza haciéndose eco de una crítica formulada en el marco del debate filosófico contemporáneo y a la que tratará de dar respuesta. De acuerdo con esta crítica, habría algo opresivo en la idea de que lo que decimos y queremos decir corrientemente, en nuestro lenguaje ordinario (what we say and mean), pueda dominar sobre lo que podemos decir y querer decir desde la filosofía. Cavell acepta que pueda parecer opresiva una filosofía que parece tener información misteriosa, extraña (uncanny, un término característicamente cavelliano) sobre algunas de nuestras asunciones filosóficas más personales: por ejemplo, acerca de la falta de certeza de nuestro conocimiento de la existencia del mundo externo y de las otras mentes; o sobre la distinción entre lo descriptivo y lo normativo, o entre cuestiones de hecho y cuestiones de lenguaje. El propósito de Cavell en este ensayo es aclarar lo que entraña, en su consideración, proceder en filosofía a partir de lo que decimos y queremos decir con nuestras palabras más familiares, con nuestro lenguaje ordinario, para mostrar que, lejos de ser opresiva, esa filosofía es iluminadora y ayuda a superar formas de alienación ligadas a la falta de conciencia de nuestro propio lenguaje cotidiano.

Tomando como hilo conductor una discusión detenida y en profundidad de las afirmaciones críticas de Benson Mates (en su ensayo On the verification of statements about ordinary language), Cavell consigue delinear su propia posición al mismo tiempo que defiende la filosofía del lenguaje ordinario. Lo hace identificando una forma errónea de interpretar esa reivindicación, a la que llama el problema de la "normatividad": la doble idea de que (1) las proferencias descriptivas se oponen a las normativas, y de que (2) las proferencias prescriptivas son ejemplos típicos de proferencias normativas. Que esta doble idea se apoya en una mala comprensión se muestra porque las enunciaciones descriptivas no se oponen, según afirma, a las normativas, sino que las presuponen: "no podríamos hacer eso que llamamos describir si el lenguaje no proporcionara (no se nos hubieran enseñado) formas normativas de describir" (p. 65). Contrapuestamente, si una proferencia normativa es aquella que se emplea para establecer reglas o estándares, las proferencias prescriptivas no pueden ser ejemplos de proferencias normativas. Pues al establecer una nueva regla no decimos cómo deberíamos realizar una acción, sino cómo esa acción se lleva a cabo o cómo puede llevarse a cabo. 
Esta sutil disolución de la distinción entre lo normativo y lo descriptivo permite a Cavell introducir la noción original de declarativo categórico (descripción-regla, o descripción que establece una regla diciendo cómo se realiza una acción), en lo que constituye una revisión explícita de la noción de Kant de imperativo categórico. En la interpretación de Cavell, el imperativo categórico kantiano puede entenderse como un declarativo categórico, pues describe "qué es comportarse moralmente". El declarativo categórico no establece lo que debe hacerse cuando se quiere actuar moralmente, sino que describe (al menos parte de) lo que hacemos cuando actuamos moralmente.

La misma perspectiva puede generalizarse, propone Cavell, cuando se consideran enunciaciones relativas a lo que debemos hacer o decir. Estas enunciaciones sólo cobran sentido en un contexto en el que sabemos que estamos, de hecho, haciendo o diciendo algo, pero que lo estamos haciendo o diciendo incorrectamente, de manera inapropiada, irreflexivamente, etc. Y esta apreciación es igualmente válida cuando nuestras enunciaciones tratan de lo que deberíamos hacer, o podemos o tenemos que hacer, o se espera que hagamos, o estamos autorizados a hacer, y otras expresiones modales análogas. Todos estos casos sugieren, en la valoración de Cavell, que nuestras proferencias de este tipo no están prescribiendo una nueva acción, sino estableciendo la regla de acción que antecede y es pertinente para juzgar o valorar lo que en ese momento estamos haciendo o diciendo - $-\mathrm{O}$, añade Cavell, para lo que somos-. El ejemplo wittgensteiniano del juego del ajedrez le sirve para mostrar de una forma más clara su punto de vista: cuando decimos "la reina se mueve (siempre, de hecho) en línea recta", o "la reina se puede mover en línea recta", o "hay que mover la reina en línea recta", no hay aquí un salto desde un "es" a un "debe ser", sino una mera apreciación de cuál de estas cosas deberían decirse en una situación concreta y cuándo; por ejemplo, teniendo en cuenta la posición o las circunstancias en las que se encuentra la persona que juega. La conclusión de Cavell es que, en general, las enunciaciones que incluyen imperativos y otras expresiones modales como debe, puede, debería, tendría, etc. requieren del trasfondo de una acción ya establecida (o, se añade aquí, del trasfondo de una práctica ya establecida) en cuyo marco se habla de la acción concreta que se realiza en el momento.

La significación de estos declarativos categóricos por medio de los cuales, a partir de los usos disponibles en el lenguaje ordinario, establecemos una regla o norma, reside en que nos permiten recordar o enseñar cuáles son las implicaciones prácticas de nuestras proferencias. Estas implicaciones prácticas las entiende Cavell como lo que hacemos explícito en aclaraciones del tipo "Cuando deci- 
mos... implicamos...". Por ejemplo (y es el ejemplo del que parte la discusión con Mates), el uso de la expresión "voluntariamente" implica que la acción que se valora o juzga es de un tipo por el que cabe atribuir responsabilidad a la persona que actúa, por lo general en contextos en los que esa actuación merece alguna censura o reproche. Estas implicaciones prácticas de nuestras proferencias no tienen una naturaleza semántica, sino que pertenecen a la gramática de su uso.

Esta distinción entre lo que se dice con una proferencia y lo que con ella se implica permite a Cavell dar respuesta a la pregunta que da título al ensayo, y que en una primera lectura podía resultar misteriosa y paradójica. La extrañeza desaparece cuando se tiene en cuenta que cuando decimos algo, con lo que decimos estamos significando además (meaning, queriendo decir) aquello que en la gramática de nuestra proferencia queda establecido como las implicaciones prácticas de decir eso que se dice. Este querer decir (implicar en sentido práctico) que va unido a lo que decimos está dado con la gramática del uso ordinario del lenguaje. Es interesante observar aquí tanto la proximidad de esta noción de Cavell con la noción de H.P. Grice de implicatura pragmática, que él mismo reconoce, como lo que le aleja del marco griceano y que él mismo hace explícito (v. notas 29, 31 y 32): la cuestión para Cavell es referente al significado pragmático de una palabra en general, no relativo a lo que ha querido decir con ella la persona que la usa en una ocasión particular.

Para Cavell, lo que una palabra significa en una lengua natural es distinto de, y no puede esclarecerse a partir de, los procesos mentales que experimenta una persona cuando habla; pues las cuestiones relativas al significado sólo pueden entenderse, como lo entendía el último Wittgenstein, cuando esa palabra se ha situado en su "entorno natural". La conciencia filosófica del lenguaje ordinario es iluminadora porque el lenguaje cambia con los cambios de la cultura que integra; y "eso es lo que explica cómo el lenguaje por el que transitamos cada día puede contener tesoros no descubiertos" (p. 89). La extrañeza de las palabras más familiares se manifiesta, podría concluirse, mientras carecemos de suficiente conciencia filosófica respecto a cómo nuestros usos de las palabras se integran en el entorno natural de una forma de vida cambiante.

Esta tarea, el esfuerzo por avanzar en una conciencia filosófica que ilumine la coherencia de nuestro lenguaje cotidiano desde nuestra forma de vida (y de nuestra forma de vida desde nuestros usos cotidianos del lenguaje), habría sido inaugurada y llevada a cabo de una manera ejemplar por el último Wittgenstein. Frente a otras formas de aproximación al lenguaje ordinario, Wittgenstein muestra la orientación de esta filosofía a través de una formulación nítida y directa, que 
Cavell cita más de una vez: "lo que nosotros hacemos es reconducir las palabras desde su uso metafísico a su uso cotidiano" (Investigaciones Filosóficas, \116). Esta tarea, observa Cavell, presupone que antes nos hemos dejado llevar fuera de lo cotidiano, "viviendo, por decirlo así, exiliados de nuestras palabras, en una posición en la que no estamos seguros de querer decir lo que decimos" (p. 16). Podría afirmarse que el estudio de la forma en que Wittgenstein confronta este problema, y la forma de su respuesta a él, subyacen al ensayo La accesibilidad de la filosofía del segundo Wittgenstein. De nuevo, el punto de partida es el esfuerzo por dar respuesta a un estudio bien valorado en su contexto académico contemporáneo (la obra de David Pole La segunda filosofía de Wittgenstein) y que, para Cavell, distorsiona la importancia de lo que se pone en juego en la obra de Wittgenstein.

En su Prefacio a la edición en castellano que reseñamos, el propio Cavell señala tres ideas presentes en este ensayo que, en su autocomprensión, motivaron posteriormente su obra Reivindicaciones de la razón (1979): que el poder comunicativo del lenguaje no requiere de nada más que del hecho de que compartimos y mantenemos nuestras formas de vida; que el estilo de las Investigaciones Filosóficas puede considerarse un cruce entre los géneros de la dogmática, la confesión y el diálogo; y, finalmente, que un objeto de interpretación, como lo eran las Investigaciones Filosóficas, había pasado a ser un medio de investigación. Además de estas ideas, y junto a las materias que el ensayo trata sistemáticamente, en esta reseña importa sobre todo volver sobre la tesis de Cavell que hemos enunciado más arriba: su idea de que la búsqueda filosófica de Wittgenstein se orienta a identificar las condiciones necesarias del mundo compartido.

Frente a una mala comprensión de la filosofía wittgensteiniana, que le atribuye alguna tesis sustantiva sobre el conflicto entre el lenguaje ordinario y el lenguaje filosófico, la lectura de Cavell invita a considerar que la filosofía no entra en conflicto con el lenguaje cotidiano cuando niega aquello que sabemos o tenemos por verdadero (como la existencia del mundo exterior, etc.), sino debido a su esfuerzo por escapar a las formas de vida que son lo único que puede proporcionar coherencia a la expresión de lo sabido o tenido por verdadero en nuestro lenguaje más cotidiano. El hecho de que Cavell hable de formas de vida en plural conlleva ya una determinada comprensión de ellas: "compartimos líneas de intereses y sentimientos, modos de respuesta, sentidos del humor y de la significación y de la satisfacción, de qué es monstruoso, de cuándo una expresión es una aseveración, cuándo una apelación, y cuándo una explicación: todo el torbellino del organismo que Wittgenstein llama 'formas de vida"' (p. 99). Este entramado proporciona el marco que permite que, cuando aprendemos y enseñamos palabras en ciertos 
contextos, seamos capaces de proyectarlas en otros contextos nuevos - y sin que nada asegure el éxito en esa proyección, más allá del marco compartido.

Esta comprensión del modo en que se articula la expresión de nuestro conocimiento con nuestras formas de vida fuerza a preguntarse por el tipo de conocimiento que consiste en saber lo que nuestras palabras cotidianas significan, en conocer lo que corrientemente significamos al usar una expresión, o cuáles son las circunstancias concretas en que una expresión se usa de hecho. Frente a otras posiciones filosóficas, que considerarían que este conocimiento del lenguaje cotidiano sólo puede ser conocimiento empírico — puesto que sería, suponen, conocimiento de cuestiones de hecho-, Wittgenstein y la filosofía del lenguaje ordinario no se están preguntando simplemente, afirma Cavell, por cómo se usa el lenguaje. Se trata, más bien, de "entender algo que ya está patente ante nuestros ojos” (Investigaciones Filosóficas, §89). En este punto de su reconstrucción del pensamiento de Wittgenstein es donde Cavell articula y justifica su aproximación a Kant: pues, en su interpretación, este tipo de conocimiento es "un conocimiento de lo que Wittgenstein quiere decir con el término gramática -el conocimiento que Kant llama 'trascendental'_-” (p. 113). Al igual que el término transcendental remite, para Kant, a un conocimiento que incumbe a la posibilidad misma del conocimiento, a las condiciones que hacen posible el conocimiento en cuanto tal, lo que Wittgenstein llama gramática remite al "tipo de enunciado que hacemos sobre los fenómenos (...) Nuestra investigación es, por ello, de índole gramatical” (Investigaciones Filosóficas, 『90) Así mismo, la ilusión trascendental de que conocemos lo que trasciende a las condiciones del conocimiento posible, encontraría para Cavell su reflejo en la ilusión producida por creer que podemos conocer lo que significan las palabras prescindiendo de algún juego de lenguaje en las que su uso cobre inteligibilidad. La diferencia entre Kant y Wittgenstein es igualmente clara para Cavell. Pues, para Wittgenstein, la consideración misma de que pueda haber una 'cosa en sí' que no podemos conocer, o que sí podemos, no puede formularse inteligiblemente, al faltar el juego de lenguaje que daría sentido al a noción misma de 'conocer algo tal y como realmente es'.

El último aspecto de la lectura de Cavell sobre Wittgenstein que se destacará en esta reseña tiene que ver con el problema de la relación entre el conocimiento del lenguaje ordinario y el autoconocimiento. Una lengua natural es aquella hablada por el conjunto de sus hablantes, y hablar una lengua es una competencia práctica. Cuando alguien considera preguntas como las siguientes: ¿qué deberíamos decir en esta ocasión? O ¿en qué circunstancias llamaríamos...?, la respuesta tiene la forma de un requerimiento para que la propia persona, en tanto que hablante 
competente, describa lo que hace o dice, y por tanto que diga algo sobre sí misma. Cavell se da cuenta de la potencial paradoja que esto entraña para el método de análisis tanto de Wittgenstein como de la filosofía del lenguaje ordinario, y lo formula de manera directa: "La cuestión es: ¿por qué algunas reivindicaciones sobre mí se expresan en la forma 'nosotros (...)'?, ¿'sobre qué puedo hablar yo por los otros basándome en lo que he aprendido de mí mismo? (...) ¿Pero cómo sé que los otros hablan como yo?” (p. 114) La respuesta de Cavell abre el paso al escepticismo, al ser igualmente directa: no lo sé. Sin embargo, su conclusión va más allá: pues la anterior aparente paradoja daría plausibilidad a la idea de que la pregunta "¿cómo sabemos lo que decimos (intentamos decir, deseamos decir)?" es un aspecto de la pregunta general "¿cuál es la naturaleza del auto-conocimiento?" (p. 116).

El ensayo Problemas estéticos de la filosofía moderna sigue siendo netamente wittgensteiniano en el método de Cavell. Comienza planteando dos problemas de la estética contemporánea, para abordar a continuación ambos con las herramientas del análisis del lenguaje ordinario. Los dos problemas son: la controversia sobre si un poema, o una metáfora, pueden parafrasearse para desplegar su significado; $y$ la constatación de Cavell de que los juicios estéticos adoptan la forma del tipo de enunciaciones que caracterizan a la filosofía del lenguaje ordinario, cuando apela a lo que 'nosotros' decimos o queremos decir, o no podemos o debemos decir o querer decir. En ambos casos, sus análisis permiten iluminar aspectos que con frecuencia permanecen opacos —y, así, generan alienación.

Austin y la crítica, escrito como revisión de la obra de John L. Austin Philosophical Papers, hace explícita su deuda con el autor de Cómo hacer cosas con palabras, si bien desde una posición distanciadamente crítica. Cavell le reconoce a Austin haber ofrecido la versión más pura de lo que pasó a llamarse la filosofía de Oxford o filosofía del lenguaje ordinario. Le reprocha, sin embargo, no haber sido capaz de llegar a darse cuenta de las consecuencias que tenía para la filosofía la radicalidad de su propia propuesta. Cavell fija su atención en los "términos de crítica" empleados por Austin, y ejemplifica el proceder austiniano en el modo en que Austin critica a otros filósofos. Para Cavell, no es posible saber hasta qué punto las críticas de Austin son válidas o hasta qué punto son una proyección de su propia personalidad. Si bien este juicio sólo puede considerarse negativo en el marco de la crítica filosófica, en el Prefacio a la edición que reseñamos Cavell se esfuerza por hacer justicia a Austin, reconociendo que "en los últimos años la obra de Austin ha asumido para mí una importancia renovada” (p. 21). 
El capítulo VI está dedicado a El libro de Adler, la obra de Kierkegaard sobre autoridad y revelación. Cavell trata de acercar el pensamiento de Kierkegaard proponiendo una lectura filosófica actualizada. Para ello, una vez más, la última filosofía de Wittgenstein ofrece la perspectiva desde la que tratar de comprender la obra: si imaginar un lenguaje es imaginar una forma de vida, hablar religiosamente no es algo que tenga lugar por el mero uso de determinadas palabras o formas de expresión; es hablar desde una determinada perspectiva. Por ello, lo religioso, sugiere Cavell, "debiera entenderse como una forma de vida wittgensteiniana" (p. 237). De esta forma, el lenguaje religioso sólo puede comprenderse cuando se comparte esa perspectiva especial o forma de vida.

Conocimiento y reconocimiento (capítulo IX) es un ensayo de respuesta al libro de Norman Malcolm The privacy of experience. Cavell reconoce sus raíces en la filosofía analítica, de la que se distancia críticamente en su formulación más tradicional (el cartesianismo o el empirismo británico), al mismo tiempo que vuelve a reivindicar su adscripción a la última filosofía de Wittgenstein y la filosofía del lenguaje ordinario. Pero también es, más sorprendentemente, una reivindicación del escepticismo o, cuanto menos, de una forma de entender el escepticismo. El valor que Cavell atribuye a esta posición filosófica no es, sin embargo, el de ser una posición sustantiva sobre el conocimiento o sobre el mundo: "el conocimiento del escéptico, si sentimos su fuerza, result[a] devastador: no desafía una creencia particular o conjunto de creencias acerca de, digamos, las otras mentes; el escéptico desafía la base de nuestras creencias en general, nuestra capacidad de creer en absoluto" (p. 314). Esta perspectiva escéptica entra en directo diálogo con la que Cavell adscribe a Wittgenstein y la filosofía del lenguaje ordinario. Pues, de acuerdo con ella, las cuestiones sobre las que las diversas posiciones filosóficas entran en conflicto entre sí, o con el sentido común, no son las creencias que se tienen acerca del mundo. Creencias del tipo de que existe el mundo, o que existen las personas en él, son el fundamento sobre el cual se apoyan cualesquiera otras creencias que podamos tener sobre el mundo o sobre las personas que hay en él.

Incluso si la interpretación que Cavell ofrece sobre el conocimiento escéptico resulta original y heterodoxa, es fácil reconocer en ella la idea wittgensteiniana de que determinadas declaraciones son gramaticales: no se refieren a cuestiones de hecho que puedan contrastarse empíricamente, sino que expresan el modo en que usamos nuestros conceptos y fundan la significación misma de nuestras descripciones. De este modo, el propio Wittgenstein no habría podido (ni habría pretendido) sustraerse al valor de la pregunta escéptica. 


\section{CONSIDERACIONES FINALES}

La obra ¿Debemos querer decir lo que decimos? es, en su conjunto, una lectura difícil. La variedad de cuestiones que Cavell plantea en cada ensayo, unida a la minuciosidad y los matices de sus análisis, no van acompañados de una presentación sucinta del orden en que se desarrollarán las cuestiones ni, en general, de una formulación suficientemente explícita y precisa de sus tesis y puntos de vista. Tampoco hay en sus ensayos unas conclusiones que ayuden a reconstruir el discurso desde un punto de vista argumentativo. El estilo de escritura de Cavell es wittgensteiniano, y no ortodoxamente académico: alcanza una profundidad iluminadora en muchos de sus análisis, sin detenerse después en dar forma a las consecuencias que podrían seguirse. Su estilo es el de un discurso inconcluso y abierto, más sugerente que afirmativo, más crítico que propositivo. Hablando del último Wittgenstein, Cavell sugiere que su estilo es, sobre todo, el de la confesión y el diálogo. En la confesión, entendida como auto-testimonio, como expresión del auto-conocimiento, no se trata de explicar o justificar, sino de describir lo que uno conoce de sí, y de quienes pueden identificarse con uno. El mismo carácter de auto-expresión de un conocimiento de sí que puede ser, y en ocasiones logra ser, un conocimiento mutuo en una forma de vida compartida, se encuentra en la escritura y el pensamiento de Cavell.

El valor del diálogo para el pensamiento y la escritura es, sin embargo, mucho más explícito y claro en Cavell de lo que lo es en Wittgenstein. Pues, como ha habido ocasión de poner de manifiesto en esta reseña (y como se pone de manifiesto en muchísimas más ocasiones a lo largo del volumen), Cavell entra en un diálogo real con interlocutores (y antagonistas) reales, incluidos algunos críticos de sus propios escritos o de los autores a los que respeta y valora. Cavell parece escribir motivado por un sentimiento de urgencia, o por su sentido de la responsabilidad, ante la necesidad de dar respuesta a lo que considera malas comprensiones, o erróneas representaciones de esas posiciones filosóficas que estima, o de la propia. Esto podría explicar por qué la mayoría de sus ensayos comienzan con una referencia a algún otro escrito, y el desarrollo de su discusión cobra la forma de un diálogo, o una crítica metódica y ponderada dirigida hacia ese escrito, en un esfuerzo explícito por restaurar un punto de vista correcto o justo sobre la cuestión tratada.

La lectura del conjunto de ensayos sugiere dos posibles objeciones. La primera consideraría que el virtuosismo que Cavell alcanza en sus análisis de nuestro lenguaje más cotidiano y familiar no le permite, sin embargo, llegar a establecer una posición filosófica propia suficientemente definida. La respuesta que Cavell podría dar a esta objeción tendría en cuenta, sin duda, sus numerosas reflexiones 
sobre el valor del análisis del lenguaje ordinario para iluminar aspectos no sólo de lo que decimos y significamos (queremos decir, implicamos prácticamente) con nuestras palabras, sino de nuestras formas de vida entretejidas con esas palabras. Sobre todo, Cavell podría responder poniendo en valor la importancia de contribuir así a superar la alienación causada por nuestra falta de conciencia respecto a nuestro uso del lenguaje. En relación con su método de análisis y la presunta falta de una posición filosófica sustantiva ligada a él, Cavell podría recordar su propia afirmación de que "La filosofía del lenguaje ordinario versa sobre cualquier cosa que verse el lenguaje ordinario" (p. 20).

Una segunda objeción podría considerar que la capacidad de ponderación en la crítica que Cavell muestra poseer de manera egregia aparece extrañamente ausente cuando se trata del último Wittgenstein. No sólo las numerosas apelaciones a su pensamiento, sino el grado de identificación que se entrevé en su aplicación de la filosofía wittgensteiniana, podrían hacer pensar en una falta de distancia crítica que sesgaría su interpretación sobre el último Wittgenstein. La respuesta que quizá no daría Cavell, pero que puede proponerse aquí, sería la siguiente. A lo largo de la presente reseña se ha puesto de manifiesto que la lectura que Cavell hace del último Wittgenstein no es ortodoxamente académica, ni se ajusta a alguna de las interpretaciones disponibles más influyentes. Por el contrario, su aproximación es personalísima e indudablemente original. Tanto la lectura kantiana que Cavell ofrece del último Wittgenstein, como su énfasis en el problema del autoconocimiento en tanto que unido al método de análisis wittgensteiniano (“¿sobre qué puedo hablar yo por los otros basándome en lo que he aprendido de mí mismo?”), y así mismo el desvelamiento de un carácter testimonial en sus escritos, difícilmente podrían dejar de valorarse como una propuesta retadora en su originalidad y lucidez.

Es preciso dedicar unas últimas palabras a la edición y la traducción del volumen. La actualidad y pertinencia de las cuestiones que trata Cavell no sólo justifican esta edición en castellano, sino que merecen elogio en unos tiempos difíciles para la filosofía en general. La traducción consigue trasladar al castellano, con fidelidad y acierto, el estilo de Cavell, que aúna la calidad literaria de su escritura con un tono familiar y cercano.

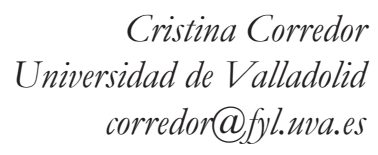

